Energy and Sustainability VIII 163

\title{
DISTANCE MEASURES FROM REPLICATOR SYSTEMS WITH NONLINEAR PAIRWISE INTERACTIONS FOR ENVIRONMENTAL RISK MANAGEMENT
}

\author{
YURI A. PYKH ${ }^{1} \&$ IRINA G. MALKINA-PYKH ${ }^{2}$ \\ ${ }^{1}$ St. Petersburg Mathematical Society, Russia \\ ${ }^{2}$ St. Petersburg State Institute of Psychology and Social Work, Russia
}

\begin{abstract}
Replicator dynamics is an evolutionary strategy well established in different disciplines of environmental sciences. It describes the evolution of self-reproducing entities called replicators in various independent models of, for example, genetics, ecology, prebiotic evolution, and socio-biology. So, the replicator systems arise in an extraordinary variety of ecosystem modelling situations. In this report we combine the three universalisms in modelling: nonlinear pairwise interactions concept, dynamical systems theory and generalized relative entropy as a distance measure for environmental risk management. We introduce two hypotheses concerning the structure and types of properties of the system's entities' nonlinear interactions and derive generalized replicator dynamic equations. If there exists a nontrivial equilibrium point for a generalized replicator system then we construct an entropy-like Lyapunov-Meyer function (LMF) for this system and prove that it is a relative entropy function or the function of information divergence. We have proven that negative relative entropy is a convex function for a probability space and receive new distance measures (divergence) between two probability distributions. In conclusion, we show, as an example, that relative entropy as distance measures may be used as sustainability indicators for estimating the efficiency energy use of wastewater treatment. These results allow us to suggest that these new distance measures can be applicable to a wide set of real environmental situations.
\end{abstract}

Keywords: nonlinear pairwise interactions, replicator dynamics, Lyapunov functions, distance measure, divergence, sustainable development.

\section{INTRODUCTION}

Sustainable development is a complicated concept that has arisen to synthesize ideas that simultaneously address the risk of environmental impacts and the needs of people. Sustainable development commonly is defined as the process of meeting the needs of the present generation without compromising the ability of future generations to meet their needs. This concept arose in the late 1980s as an approach to balance economics and environment. Sustainable development embodies several advancements over other models that have characterized the development process. One advancement is drawing distinctions between the growth component and the efficiency component of development. It is evident that measures of divergence between two points play a key role in many environmental problems. One can find descriptions of energy, water and agricultural aspects of sustainable development in the books [1]-[3].

Mathematical models are useful tools for both understanding the dynamics of populations and impact assessment for several reasons. In the first place, models can lead to an increase of the general understanding of a system and help generate hypotheses and direct field experiments. In the second place, models can be used as a tool for evaluating alternative plans (scenarios) or by providing rules of thumb to policy makers.

Ecosystem stability and the response of ecosystems to disturbance are of crucial importance for conservation management. A variety of ecological interpretations have been given to the term "stability". The most generally used concept refers to the stability in the 
vicinity of an equilibrium point in a deterministic system. Pimm [4] distinguished two aspects of this stability: "resilience", the speed at which a system returns to its equilibrium following a perturbation, and "resistance", the ability of a system to withstand displacement by a disturbance in its environment. These two properties are usually held to be responsible for the persistence of ecosystems in an unpredictable environment. A "stable" ecosystem might be expected to exhibit both of these properties to some degree. The linkages between resilience and the stability of dynamical systems are discussed, along with its role in understanding of the evolution of such systems.

Many problems of investigating stability and calculating characteristics of dynamical systems (time and value of transient processes, speed of convergence, stability region, integral criteria, etc.) are solved using the direct Lyapunov method. The method is based on searching the function having certain properties along the system solutions. See, for example Common and Perrings [5].

The primary goal of this paper is to study the implications of Lyapunov functions theory for the analysis of nonlinear pairwise interactions replicator systems. We build Lyapunov-Meyer functions as entropy-like functions (distance measures). The existence of the Lyapunov-Meyer functions allows us to construct practically all known entropy and relative-entropy functions. Our investigations are based mainly on the stability postulate stated by Chetaev in 1936 [6].

Stability postulate. Stability, which is a fundamentally general phenomenon, apparently, must manifest itself in basic laws of nature in some way. If knowledge is constructed from the requirement of small deviations from nature, then scientific thinking must (or can) rely on some Lyapunov function. Certainly, this function always exists according to the stability postulate.

Many years later in 1967 stability postulate, in more narrow sense, was used by Coleman and Mizel in their famous paper "Existence of Entropy as a Consequence of Asymptotic stability" [7].

In 1968, this postulate was stated mathematically by Meyer [8], who proved that, for dynamical systems whose limit sets consist of only isolated rest points or cycles, i.e., for Morse-Smale systems, there always exists a Lyapunov function, which increases on the set of wandering points of the system. Meyer, followed by Smale [9], suggested the term energy functions for such functions; however, it is more natural and convenient to refer to them as Lyapunov-Meyer functions. One of the best-known examples of such function is entropy in Boltzmann's H-theorem.

Prigogine [10] first pointed out the importance of the relationship between Lyapunov functions and entropy: "The positive time direction is associated with the increase of entropy. Let us emphasize the strong and very specific way in which the one-sidedness of time appears in the second law. According to its formulation it leads to the existence of a function having quite specific properties as expressed by the fact that for an isolated system it can only increase in time. Such function plays an important role in modern theory of stability as initiated by the classic work of Lyapunov. For this reason they are called Lyapunov functions (or functional).

The entropy $\mathrm{S}$ is a Lyapunov function for isolated systems. As shown in all textbooks, thermodynamic potentials such as the Helmholtz or Gibbs free energy are also Lyapunov functions for other "boundary conditions" (such as imposed values of temperature and volume). In all these cases the system evolves to an equilibrium state characterized by the existence of a thermodynamic potential. This equilibrium state is an "attractor" for nonequilibrium states. 
For dynamical systems arising from physics the Lyapunov functions will typically have a thermodynamic interpretation (energy, entropy etc.) but its origin is not evident.

In an attempt to show how such an interpretation this idea might proceed, we analyze below a class of macrosystems with nonlinear pairwise interactions for with an assumption of structure and type of interactions has consequences that there exist entropy-like functions.

\section{EQUATIONS OF MACROSYSTEMS WITH NONLINEAR PAIRWISE INTERACTIONS}

One of the important assumptions in classic Lotka-Volterra and replicator systems is that the functional responses are linear functional responses. Usually additive linear models for predicting combined interactions effects cannot account for non-linearities in combined functional response introduced by non-trophic interactions Ayala et al. [11]. Exponential and power law responses were used to model social dynamics by Beechman and Farnsworth [12]. The PhD thesis by Merrifield [13] provides a review on the concept of a force including nonlinear in different interacting systems in Chapter 5.

Consider a macrosystem formed by a sufficiently large number $N$ of interacting objects. The classical definition of macrosystems is as follows: these are systems in which a chaotic behavior at the microlevel transforms into a deterministic behavior at the macrolevel. Suppose that, at a moment $t$, the macrosystem under consideration contains $n$ different types of objects and the number of objects of type $i$ is $x_{i}(t)$, where $i=1, \ldots, n$ and $\sum_{i=1}^{n} x_{i}(t)=N(t)$. Consider the relative numbers $p_{i}(t)=x_{i}(t) / N(t), p_{i}(t)$ of various types' objects. Obviously, $\quad \sum_{i=1}^{n} p_{i}(t)=1, \quad$ i.e., vector $\mathrm{p}(t) \in \sigma_{p}^{n}=\left\{\mathrm{p} \in \mathbb{R}^{n}: p_{i} \geq 0, i=1,2, \ldots, n, \mathbf{e}^{T} \mathrm{p}=1\right\}$, where $\sigma_{p}^{n}$ is the standard simplex in Euclidean $n$-space $\mathbb{R}^{n}$ and, $\mathbf{e}$ is the vector of ones. Thus, the state of such a macrosystem at each moment $\mathrm{t} i$ determined by the vector $\mathrm{p}(t)=\left(p_{i}(t), \ldots, p_{n}(t)\right)$.

We consider the systems in which only pairwise interactions occur during a short time interval $(t, t+\Delta t)$; in other words, those systems in which simultaneous interactions of more than two particles are impossible.

We make the following two assumptions about interactions between objects in a macrosystem [14].

Hypothesis 1: The interaction between objects of types $i$ and $j$ is characterized by the so-called interaction strength, which we denote by $w_{i j}$ and regard as a quantitative characteristic of the effect of the interaction between two objects of types $i$ and $j$ on the rate of change of the relative number $p_{i}(t)$ of objects of type $i$.

Remark 1: Apparently, the notion of an interaction strength first appeared in mathematical ecology. A fairly detailed study of this notion and a survey of related results are contained in Ayala et al. [11].

Remark 2: Note that the definition given above does not imply that $w_{i j}=w_{j i}$. We also emphasize that the asymmetry of results of interaction plays an essential role in many cases [15].

Hypothesis 2: For each macrosystem under consideration, there exists a set of probability distribution functions $f_{i}\left(p_{i}\right)$, where $i=1, \ldots, n$, which determine the probability of the 
interaction of each object of type $i$ with any other object in the macrosystems. Thus, the probability of the pairwise interaction between objects of types $\mathrm{i}$ and $\mathrm{j}$ is determined by the product $f_{i}\left(p_{i}\right) f_{i}\left(p_{i}\right)$.

Remark 3: The probability distribution functions $f_{i}$ (nonlinear response functions) obeys the conditions $f_{i}(0)=0, \quad \partial f_{i} / \partial p_{i}>0$ for $\quad p_{i}>0, \quad \partial f_{i} / \partial p_{i} \geq 0$ for $\quad p_{i}=0$ and $f_{i}(1)=1[16]$.

Note that the standardization condition for response functions was investigated in the article by Pykh and Malkina-Pykh [17].

It follows from the two hypotheses stated above that, for small $\Delta t$, the dynamics of the system is determined by the relation:

$$
p_{i}(t+\Delta t)=p_{i}(t)+\Delta t \sum_{j=1}^{n} w_{i j} f_{i}\left(p_{i}(t)\right) f_{j}\left(p_{j}(t)\right)+o(\Delta t),
$$

subject to the constraints $\sum_{i=1}^{n} p_{i}(t)-1=0$, and $f_{i}(0)=0$, for $i=1,2, \ldots, n$ which ensure the invariance of the simplex $\sigma_{p}^{n}$ and all of its faces. Using an approach common in analytic mechanics, we treat the system under consideration as a nonfree system subject to the ideal holonomic constraints:

$$
f_{i}\left(p_{i}\right)\left(\sum_{i=1}^{n} p_{i}(t)-1\right)=0, \quad i=1, \ldots, n
$$

and apply the principle of replacing constraints by reaction forces to this system [18]. Passing to the limit as $\Delta t \rightarrow 0$ and taking into account the obvious identity $\sum \dot{p}_{i}=0$ for the sum of derivatives, we obtain the following equation for the constraint multipliers (the indeterminate Lagrange multipliers) $\lambda_{i}$ :

$$
\sum_{i j}^{n} w_{i j} f_{i}\left(p_{i}\right) f_{j}\left(p_{j}\right)-\sum_{i=1}^{n} \lambda_{i} f_{i}\left(p_{i}\right)=0
$$

This equation has two obvious solutions, the trivial solution $\lambda_{i}=\sum_{j=1}^{n} w_{i j} f_{j}\left(p_{j}\right)$ and the nontrivial solution:

$$
\lambda_{i}=\lambda=\frac{\sum_{i j}^{n} w_{i j} f_{i}\left(p_{i}\right) f_{j}\left(p_{j}\right)}{\sum_{i=1}^{n} f_{i}\left(p_{i}\right)}, \quad i=1, \ldots, n
$$

Using the nontrivial solution and denoting the sum $\sum_{i=1}^{n} f_{i}\left(p_{i}\right)$ by $\theta(\mathrm{p})$, we obtain the following system of differential equations determining the evolution of the probability distribution $\mathrm{p}(t)$ :

$$
\dot{p}_{i}=f_{i}\left(p_{i}\right)\left(\sum_{j=1}^{n} w_{i j} f_{j}\left(p_{j}\right)-\theta^{-1}(\mathrm{p}) \sum_{i j}^{n} w_{i j} f_{i}\left(p_{i}\right) f_{j}\left(p_{j}\right)\right), \quad i=1, \ldots, n .
$$


This system of equations was first obtained from balance considerations in Pykh [19] without the use of the Lagrange method. In what follows, it is convenient to pass to the matrix form. System (1) is written in this form as:

$$
\dot{\mathrm{p}}=D(\mathrm{f})\left(\mathrm{Wf}-\mathbf{e} \theta^{-1}(\mathrm{p})\langle\mathrm{f}, \mathrm{Wf}\rangle\right) .
$$

Here, $\mathrm{f}(\mathrm{p})$ is the vector $\left(f_{1}\left(p_{1}\right), \ldots, f_{n}\left(p_{n}\right)\right) ; \quad D(\mathrm{f})=\operatorname{diag}\left(f_{1}, f_{2} \ldots, f_{n}\right)$; $\mathrm{W}=\left(w_{i j}\right)$ is the matrix of interactions; and $\theta(\mathrm{p})=\langle\mathbf{e}, \mathrm{f}(\mathrm{p})\rangle$, where $\langle\cdot, \cdot\rangle$ denotes inner product. Obviously, since $\langle\dot{\mathrm{p}}(t), \mathbf{e}\rangle \equiv 0$ and $f_{i}(0)=0$ it follows that the simplex $\sigma_{p}^{n}$ and each of its faces are invariant sets for system (2). Note that system (2) determines the dynamics of objects with nonlinear pairwise interactions; the matrix $\mathrm{W}$ determines the structure of interactions, and the response functions determine their types. The elements of the matrix $\mathrm{W}$ are generalized interaction strengths, i.e., by analogy with the thermodynamics of irreversible processes, are "reasons" causing changes in the speed of flows.

Let us rewrite eqn (2) as:

\section{ESCORT DISTRIBUTIONS}

$$
\dot{\mathrm{p}}=\theta D(\mathrm{f})\left(\mathrm{Wf} \theta^{-1}-\mathbf{e E}(\mathrm{p})\right),
$$

where $E(p)=\theta^{2}(p)\langle f, W f\rangle$. Using the terminology of the theory of neural networks, we refer to $E(p)$ as the energy function of the macrosystem under consideration. Note that in the population genetic theory this function named as population fitness [20], and in the evolutionary game theory as utility function [21]. System (3) and the energy function $\mathrm{E}(\mathrm{p})$ naturally determine the introduction of new additional variables:

$$
x_{i}(\mathrm{p})=f_{i}\left(p_{i}\right) \theta^{-1}(\mathrm{p}) \quad i=1, \ldots, n .
$$

Obviously, $\quad \mathrm{x}=\left(x_{1}, x_{2} \ldots, x_{n}\right) \in \sigma_{x}^{n}=\left\{\mathrm{x} \in \mathbb{R}^{n}: x_{i} \geq 0, \mathrm{e}^{\mathrm{T}} \mathrm{x}=1\right\}$ for $\mathrm{p} \in \sigma_{p}^{n} . \quad$ The indices $x$ and $p$ are used in the notation of simplexes in order to avoid confusion. Consider change (4) in more detail. If this is a diffeo-morphism, then it can be regarded not only as a simplifying change of variables customary in the theory of differential equations but also as the definition of a set of quantities with particular physical meaning.

Theorem 1. [22]: Under the conditions (remark 3) used for the response functions $f_{i}$, for $\mathrm{p} \in \sigma_{p}^{n}$, there exists a one-to-one inverse mapping to (4), which is defined by:

$$
p_{i}=f_{i}^{-1}\left(x_{i}\right) / \sum_{j=1}^{n} f_{j}^{-1}\left(x_{j}\right), \quad i=1, \ldots, n,
$$

where $f_{i}^{-1}(\cdot)$ denotes the function inverse to $f_{i}(\cdot)$. 
Remark 4: Recall that the notation $f_{i}^{-1}(\cdot)$ is used for both functions inverse in the sense of function theory and functions inverse in the algebraic sense. It is always clear from the context what is meant.

\section{EQUILIBRIUMS}

To go further, we need the following assertion [22]:

Statement 1: System (1) is invariant with respect to the replacement of the interaction matrix $\mathrm{W}$ by a perturbed matrix $\mathrm{W}_{\zeta}=\left(\mathrm{W}+\mathrm{e} \zeta^{T}(\mathrm{p})\right)$, where the components of the vector function $\zeta(\mathrm{p})=\left(\zeta_{1}(\mathrm{p}), \ldots, \zeta_{n}(\mathrm{p})\right): \sigma_{p}^{n} \rightarrow \mathbb{R}^{n}$ are bounded on $\sigma_{p}^{n}$.

We proceed to consider questions related to the existence of a nontrivial equilibrium point $\hat{\mathrm{p}} \in \operatorname{Int} \sigma_{p}^{n}$ for system (1). In Pykh [23], it was proved that $\hat{\mathrm{p}}>0$ exists if and only if all components of the vector $\mathrm{W}^{-1} \mathrm{e}$ are of the same sign. It is clear already from this result that properties of the inverse interaction matrix play a substantial role in the evolution of macrosystems.

Statement 1 implies that the coordinates of a nontrivial equilibrium point remain invariable $\forall \zeta \in \mathbb{R}^{n}$. So we have:

Statement 2. [22]: If system (4) has a nontrivial equilibrium point $\hat{\mathrm{p}} \in \operatorname{Int} \sigma_{p}^{n}$, then:

$$
\frac{\hat{\mathrm{f}}}{\langle\hat{\mathrm{f}}, \mathrm{e}\rangle}=\frac{\mathrm{W}^{-1} \mathrm{e}}{\left\langle\mathrm{e}, \mathrm{W}^{-1} \mathrm{e}\right\rangle}=\frac{\mathrm{W}_{\zeta}^{-1} \mathrm{e}}{\left\langle\mathrm{e}, \mathrm{W}_{\zeta}^{-1} \mathrm{e}\right\rangle}=\hat{\mathrm{x}} \quad \forall \zeta(\mathrm{p}) \in \mathbb{R}^{n}
$$

\section{ENTROPY-LIKE LYAPUNOV-MEYER FUNCTIONS}

Theorem 2. [22]: If system (1) has a nontrivial equilibrium point $\hat{\mathrm{p}} \in \operatorname{Int} \sigma_{p}^{n}$ and the matrix $\left(\mathrm{W}_{\zeta}^{\mathrm{T}}+\mathrm{W}_{\zeta}\right), \forall \zeta \in \mathbb{R}^{n}$ has $n-1$ negative characteristic numbers, then the function:

$$
H(\mathrm{p})=\sum_{i=1}^{n} \int_{\hat{p}_{i}}^{p_{i}} \frac{\hat{f}_{i} d x}{f_{i}(x)}+C
$$

where $C$ is a constant, is a Lyapunov-Meyer function for system (1) in $\operatorname{Int} \sigma_{p}^{n}$, and the energy function $E(\mathrm{p})$ of the system attains its maximum value $E(\hat{\mathrm{p}})$ as $\mathrm{t} \rightarrow \infty$.

It is obvious that function $H(\mathrm{p})$ depends from interaction matrix $\mathrm{W}$ only through $\hat{\mathrm{p}}$ and its characteristic numbers. It means that for each $H(\mathrm{p})$ we have a set of different systems (1) and can use this function for any two points $\mathrm{p}(t), \hat{\mathrm{p}} \in \operatorname{Int} \sigma_{p}^{n}$.

In the next section we use Theorem 2 to construct entropy characteristics and "distances" between probability characteristics. First, we note that, if $G: \mathbb{R}^{1} \rightarrow \mathbb{R}^{1}$ is a monotonically increasing smooth function and $C_{1}>0, C_{2}>0$ and $C_{3}$ are the numbers, then the function:

$$
H_{G}=C_{1} G\left(C_{2} H(\mathrm{p})\right)+C_{3}
$$


is also a Lyapunov-Meyer function for system (1).

\section{DISTANCE MEASURES}

Following Shannon information measure let us introduce $S(\mathrm{p})$ as negative entropy defined by:

$$
S(\mathrm{p})=-H(\mathrm{p})=-\sum_{i=1}^{n} \int_{\hat{p}_{i}}^{p_{i}} \frac{\hat{f}_{i} d x}{f_{i}(x)}, \quad p \in \sigma_{p}^{n} .
$$

It is easy to see, that:

$$
\lambda \sum_{i=1}^{n} \int_{\hat{p}_{i}}^{p_{i}} d x=\lambda \sum_{i=1}^{n}\left(p_{i}-\hat{p}_{i}\right) \equiv 0 \quad \forall p, \hat{p} \in \sigma_{p}^{n}
$$

and we clearly have the following relationship:

$$
S(\mathrm{p})=-\sum_{i=1}^{n} \int_{\hat{p}_{i}}^{p_{i}}\left(\frac{\hat{f}_{i} d x}{f_{i}(x)}+\lambda\right) d x \quad \forall \lambda \in \mathbb{R}^{1} .
$$

Since, by the evident equality $\partial S(\mathrm{p}) / \partial p_{i}=0$, with $p=\hat{p}$, we have that $\lambda=-1$. It is clear, that $\lambda$ is a type of Lagrange multiplier. Therefore:

$$
S(\mathrm{p})=\sum_{i=1}^{n} \int_{\hat{p}_{i}}^{p_{i}}\left(1-\frac{\hat{f}_{i}}{f_{i}(x)}\right) d x \quad p \in \sigma_{p}^{n} .
$$

The gradient of $S(p)$ with $p \in \operatorname{Int} \sigma_{p}^{n}$ is given by partial derivatives:

$$
\nabla S(\mathrm{p})=\left(1-\frac{\hat{f}_{i}}{f_{i}\left(p_{i}\right)}\right)
$$

that is strictly monotonically increasing for each $p_{i}$ and Hessian of $S(p)$ on $\operatorname{Int} \sigma_{p}^{n}$ is $\mathcal{H}(\mathrm{p})=\operatorname{diag}\left(\hat{f}_{i} \frac{\partial f_{i}}{\partial p_{i}} f_{i}^{-2}\left(p_{i}\right)\right)$. Since $\partial f_{i} / \partial p_{i}>0$ it follows that the Hessian is positive definite, and the function $S(p)$ according to definition is convex on $\operatorname{Int} \sigma_{p}^{n}$.

Another very well-known definition of convexity Jensen [24], Hardy et al. [25] is the next inequality with $p, \mathrm{q}=\operatorname{Int} \sigma_{p}^{n}$ :

$$
S(\mathrm{p})-S(\mathrm{q})-\left\langle\nabla_{q} S(\mathrm{q}), \mathrm{p}-\mathrm{q}\right\rangle \geq 0 .
$$

Evident that the expression from left-side inequality is the Bregman divergences denote by $B_{s}(\mathrm{p}, \mathrm{q})$. This name was first given the by Censor and Lent [26]. Bregman divergence or Bregman distance [27] is similar to a metric but does not satisfy the triangle inequality or symmetry. Using inequality (9) we can receive new weighted distance measure between two probability distributions: 


$$
B_{s}(\mathrm{p}, \mathrm{q})=\sum_{i=1}^{n}\left(\int_{p_{i}}^{q_{i}} \frac{\hat{f}_{i} d x}{f_{i}(x)}+\frac{\hat{f}_{i}}{f_{i}\left(q_{i}\right)}\left(p_{i}-q_{i}\right)\right) \geq 0
$$

It is obvious that $B_{s}(\mathrm{p}, \mathrm{q})$ for $\mathrm{p}=\mathrm{q}$, and $B_{s}(\mathrm{p}, \hat{\mathrm{p}})=S(p)$.

\section{LEGENDRE-DONKIN-FENCHEL TRANSFORMATIONS}

The Legendre-Donkin-Fenchel (LDF) transformation is a mathematical concept of great significance to thermodynamics, mechanics and probability theory is named after Legendre [28], Donkin [29] and Fenchel [30].

Let us denote $y_{i}=\partial S(\mathrm{p}) / \partial p_{i}$. Since $y_{i}$ is strictly monotonically increasing for $p_{i}$, this makes it clear that $y_{i}$ and $p_{i}$ are mutually reciprocal. The transformation from $p$ to $y$ is called LDF-transformation. We can find a convex function $\mathrm{Y}$ of $y$ defined by:

$$
\mathrm{Y}(y)=\sum p_{i} y_{i}-S(p) \text {. }
$$

when $y$ and $p$ are respective coordinates of the same point, and the inverse transformation from $y$ to $p$ is given by the gradient:

$$
p=\nabla \mathrm{Y}(y)
$$

Concerning the (8) we clearly have:

$$
y_{i}=\frac{\partial S(p)}{\partial p_{i}}=1-\frac{\hat{f}_{i}}{f_{i}\left(p_{i}\right)}
$$

and:

$$
p_{i}=f_{i}^{-1}\left(\frac{\hat{f}_{i}}{1-y_{i}}\right)=\frac{\partial Y}{\partial y_{i}}
$$

for $y_{i} \in\left(-\infty, 1-\hat{f}_{i}\right)$. We also denote $\partial Y / \partial y_{i}=\varphi\left(y_{i}\right)$ and after simple counting we have the following relationship:

$$
\mathrm{Y}(y)=\sum_{i=1}^{n}\left(y_{i} \varphi\left(y_{i}\right)+\int_{0}^{\varphi\left(y_{i}\right)}\left(\frac{\hat{f}_{i}}{f_{i}}-1\right) d x\right)
$$

After simple computation one can check that $\partial Y / \partial y_{i}=p_{i}$. Another form of LDFtransformation, together with (10) is:

$$
\mathrm{Y}(y)=\max _{p}(\langle p, y\rangle-S(p))
$$

Follow Amari [31] and definition (10) one can define a divergence function (distance measure) between two points $p$ and $q$ on $\sigma_{p}^{n}$ in the dual coordinates. This divergence function will be similar to the squared Riemannian distance. Additional new information on LDF-transformation is presented in Nielsen [32] and Zia et al. [33] papers. 


\section{ENTROPY CHARACTERISTICS AND DISTANCE FUNCTIONS}

Note that Theorem 1 can be used in two ways:

1. The response functions can be found for previously known entropy characteristics. Relevant examples for the Shannon and Tsallis entropy were given in Pykh [34]. It is easy to show that this approach yields response functions for all the entropy characteristics proposed in Esteban and Morales [35].

2. New entropy characteristic can be derived from some functions obeying the conditions stated for response functions. As an example, we consider the logistic function, which is widely applied in mathematical ecology and economics. This function is defined as follows:

$$
f(x)=\frac{c}{(b+c)} \frac{\left(1-e^{-\alpha x}\right)}{\left(b+c e^{-\alpha x}\right)},
$$

where $b>0, c>0, \alpha>0$.

It is obvious that $f(0)=0, f(+\infty)=c / b(b+c)$. Evaluating the corresponding integral and taking into account that arbitrary parameters can be picked in (8) (to get rid of the factors and summands), we derive the following expression for the logistic entropy $H_{l}(p)$ :

$$
H_{l}(p)=\sum_{i=1}^{n} \ln \left(1-e^{-\alpha p_{i}}\right) .
$$

Recall that $H_{l}(p)$ is a Lyapunov energy function for system (1) if and only if the conditions of Theorem 2 are satisfied and the equilibrium $\hat{p}$ is a uniform distribution; i.e. $\hat{p}=e n^{-1}$. Since all the response functions are identical in the case under study, it is necessary and sufficient that $\mathrm{W}$ be quasi-stochastic. Note that the interest in objects described by noncanonical distributions has increased noticeably over resent years [35]. It is this type of distribution that can be derived from formula (8) suggested for entropy characteristics. For further use of these characteristics, it is natural to apply Jaynes' principle of maximum information entropy [36].

Now, we underline that distance functions are defined in the case where the equilibrium distribution in system (1) is not uniform. As in the previous case, we have that new distance functions between distributions can be derived from given response functions. As before, we consider the logistic function as an example. Let the conditions of Theorem 2 be satisfied. Evaluating the integral in (8) gives the function

$$
H_{l}(p, \hat{p})=\sum_{i=1}^{n} \hat{f}_{i}\left(\frac{1-e^{-\alpha p_{i}}}{1-e^{-\alpha \hat{p}_{i}}}\right),
$$

that, by analogy, is naturally called the relative logistic entropy.

\section{ESTIMATIING OF WASTEWATE TREATMENT (EXAMPLE)}

Sustainable development is one of the great hopes for conservation and proper use of resources including biodiversity. Instead of a focus on biodiversity itself, ecosystems sustainability represents a focus on a set of ecosystems properties, especially on ecosystems resilience, persistence and integrity [37]. One of the most important 
characteristics among them is the measures of divergence between two points that plays a key role in many ecosystems' problems. As it was shown in the article of Sobczuk and Lagod on the base of the results from [37]-[38] for Wastewater Treatment Plant (WWTP) [39]: "the results of bioindication, apart from the other physical and chemical parameters, may be used in wastewater plant treatment process management". Note that authors mean there "sustainable management".

In Sobczuk and Lagod [39], the comparison of different distance measures presented in Fig. 1 demonstrates that function (11) is the best distance measure of all applied methods for estimating the efficiency of wastewater treatment. Also in Sobczuk and Lagod [39], the authors pointed out that entropy and distance measures should be appropriately chosen for complex data sets comparison. Eqn (1) with accompanying theory allows to generate distance measures according to data set character. It shows up that the distance measure (11) is best fitted to recognize differences in microbial distribution functions described there.

\section{CONCLUSIONS}

We note that the approach suggested for constructing entropy characteristics and divergences between probability distributions based on the direct Lyapunov method applied to kinetic replicator equations (i.e., equations determining the dynamics of systems with nonlinear pair interactions) provides new strategies in the macroscopic analysis of complex open objects in various areas of the natural sciences. It should also be emphasized that this approach makes it possible to introduce mixed entropy, i.e., an entropy characteristic arising when the interacting subsystems have different response functions. Due to the possibility to generate measures according to the functional form of the response function in (1) one can find the best distance measure for the certain purpose. More information about the systems with pairwise interactions can be obtained in the preprint [40].

\section{REFERENCES}

[1] Malkina-Pykh, I.G. \& Pykh, Y.A., Sustainable Energy: Resources, Technology and Planning, Computational Mechanics Publications: Southampton and Boston, 2002.

[2] Malkina-Pykh, I.G. \& Pykh, Y.A., Sustainable Water Resources Management, Computational Mechanics Publications: Southampton and Boston, 2002.

[3] Malkina-Pykh, I.G. \& Pykh, Y.A., Sustainable Food and Agriculture, Computational Mechanics Publications: Southampton and Boston, 2003.

[4] Pimm, S.L., The complexity and stability of ecosystems. Nature, 307, pp. 321-326, 1994.

[5] Common, M. \& Perrings, C., Towards an ecological economics of sustainability. Ecological Economics, 6, pp. 7-34, 1992.

[6] Chetaev, N.G., Stability and classical physical law. Proceedings of Kazan Aviation Institute, 6, pp. 3-5, 1936.

[7] Coleman, B.D. \& Mizel, V.J., Existence of entropy as a consequence of anymtotic stability. Archive for Rational Mechanics and Analysis, 25, pp. 243-270, 1967.

[8] Meyer, K., Energy functions for Morse-Smale systems. American Journal of Mathematics, 90, pp. 1031-1040, 1968.

[9] Smale, S., On Gradient dynamical systems. Annals of Mathematics, 74, pp. 199-206, 1961.

[10] Prigogine, I., Time, structure and fluctuations. Nobel lecture, 8 December 1977.

[11] Ayala, F.J., Gilpin, M.E. \& Ehrenfeld, J.G., Competition between species: Theoretical models and experimental tests. Theoretical Population Biology., 4, pp. 331-356, 1973. 
[12] Beecham, J.A. \& Farnsworth, K.D., Animal group forces resulting from predator avoidance and competition minimization. Journal of Theoretical Biology, 198, pp. 533-548, 1999.

[13] Merrifield, A.J., An Investigation of Mathematical Models for Animal Group Movement, Using Classical and Statistical Approaches. PhD doctorate, University of Sydney, 2006.

[14] Pykh, Y.A., Entropy characteristics of macrosystems with nonlinear pair interactions, Doklady Mathematics, 84, pp. 669-671, 2011.

[15] Novak, M. \& Wootton, T.J., Estimating nonlinear interaction strengths: An observation-based method for species-rich food webs. Ecology, 89, pp. 2083-2089, 2008.

[16] Pykh Y.A., Lyapunov-Meyer functions and distance measure from generalized Fisher's equations. IFAC-PapersOnLine, 48-11, pp. 115-119, 2015.

[17] Pykh, Y.A. \& Malkina-Pykh, I.G., Application of the method of double standardization for the development of environmental indices. Proceedings of the First International Conference on Environmental Indices Systems Analysis Approach (INDEX-97). EOLSS Publishers: Oxford, pp. 169-181, 1999.

[18] Loitsyanskii, L.G. \& Lur'e, A.I., Course in Theoretic Mechanics, Nauka: Moscow, 1983.

[19] Pykh, Y.A., Energy Lyapunov function for generalized replicator equations. Proceedings of International Conference "Physics and Control", pp. 271-276, 2003.

[20] Fisher, R.A., The Genetical Theory of Nature Selection, Clarendon Press: Oxford, 1930.

[21] Hofbauer, J. \& Sigmund, K., Evolutionary game dynamics. Journal of American Mathematical Society, 40, pp. 479-519, 2003.

[22] Pykh, Y.A., Escort distribution, energy and relative entropy of macrosystems with nonlinear pair interactions. Doklady Mathematics, 90, pp. 710-714, 2014.

[23] Pykh, Y.A., Equilibrium and Stability in the Models of Population Dynamics, Nauka: Moscow, 1983.

[24] Jensen, G.L.W.V., Sur les fonctions convexes et les ineqalites entre les valenrs moyennes. Acta Matematica, 30(1), pp. 175-193, 1906.

[25] Hardy, G.H., Littlewood, J.E. \& Polya, G., Inequalities, Cambridge University Press: Cambridge, 1934.

[26] Censor, Y. \& Lent, A., An iterative row-action method for interval convex programming. Journal of Optimization Theory and Applications, 34(3), pp. 321-353, 1981.

[27] Bregman, L.M., The relaxation method of finding the common points of convex sets and its application to the solution of problems in convex programming. Computational Mathematics and Mathematical Physics, 17(3), pp. 200-217, 1967.

[28] Legendre, A.M., L'intégration de quelques equations aux differences Partielles. Memoires de l'Academie Royale des Sciences, pp. 309-351, 1787.

[29] Donkin, W.F., On a class of differential equations, including those which occur in dynamical problems. Philosophical Transactions of the Royal Society of London, 144, pp. 74-113, 1854.

[30] Fenchel, W., On conjugate convex functions. Canadian Journal of Mathematics, 1, pp. 73-77, 1949.

[31] Amari, S., Differential geometry derived from divergence functions: information geometry approach. Mathematics of Distances and Applications, eds M. Deza, M. Petitjean \& K. Markov, ITHEA: Sofia, Bulgaria, pp. 9-23, 2012. 
[32] Nielsen, F., Legendre transformation and information geometry. $C I G-M E M O, \mathbf{1}(2)$, 2010.

[33] Zia, R.K., Redish, E.F. \& McKay, S.R., Making sense of the Legendre transform. American Journal of Physics, 77, pp. 614-625, 2009.

[34] Pykh, Y.A., Construction of entropy characteristics based on Lyapunov energy functions. Doclady Mathematics, 69, 355-358, 2004.

[35] Esteban, M.D. \& Morales, D.A., summary of entropy statistics. Kybernetica, 31, pp. 337-346, 1995.

[36] Jaynes, E.T., Information Theory and Statistical Mechanics. Physical Review, 106, pp. 620-630, 1957.

[37] Pykh, Y.A., Lyapunov functions as a measure of biodiversity: Theoretical background. Ecological Indicators, 2, pp. 123-133, 2002.

[38] Pykh, Y.A. \& Malkina-Pykh, I.G., Replicator equations, response functions and entropy measures in science: mathematical background. WIT Transactions on Ecosystems and Sustainable Development V, WIT Press: Southampton and Boston, pp. 5-15, 2005.

[39] Sobczuk, H. \& Lagod, G., Some distance measures for estimating the efficiency of wastewater treatment in activated sludge systems by biomonitoring. Proceedings of ECOpole, 5(2), pp. 431-436, 2011.

[40] Pykh, Y.A., Pairwise Interactions Origin of Entropy Functions. Preprint. arXiv: 1506.05731v1 [nlin.AO] 18 June 2015. 